\title{
ASSOCIAÇÃO ENTRE O NÍVEL DE ATIVIDADE FÍSICA, ÍNDICE DE MASSA CORPORALEAGILIDADE DE ESCOLARES
}

\section{ASSOCIATION BETWEEN LEVEL OF PHYSICALACTIVITY, BODY MASS INDEX AND AGILITY OF STUDENTS}

Ana Regina Leão Ibiapina Moura (Orcid: 0000-0003-0317-7322)1 Jéssica Gonçalves da Silva (Orcid:0000-0002-6495-575X)² Jorge Luiz de Brito Gomes (Orcid: 0000-0001-9852-1856) ${ }^{1}$ Ferdinando Oliveira Carvalho (Orcid: 0000-0003-0306-5910) José Fernando Vila Nova de Moraes (Orcid: 0000-0002-7394-7700)

Autor correspondente: Ana Regina Leão Ibiapina Moura E-mail: ana.ibiapina@ifma.ed.br

${ }^{1}$ Programa de Pós-graduação em Educação Física, Universidade Federal do Vale do São Francisco (Univasf).

${ }^{2}$ Graduada em Educação Física, Universidade Federal do Vale do São Francisco (Univasf).

\begin{abstract}
RESUMO
A pesquisa objetivou verificar a associação entre o nível de atividade física, o índice de massa corporal e a agilidade de adolescentes escolares da rede particular de ensino na cidade de Senhor do Bonfim/BA. Trata-se de um estudo de natureza transversal, com amostra de 75 alunos (35 meninas e 40 meninos), com idades entre 10 e 14 anos. A coleta de dados foi realizada por meio de medidas de massa corporal e estatura (para posterior cálculo do índice de massa corporal), questionário para estimativa de nível de atividade física semanal, e um teste de agilidade (teste do quadrado). A normalidade dos dados foi verificada usando o teste de Kolmogorov-Smirnov. Os dados foram analisados por meio do Teste-t de Student, ANOVA de uma entrada com post hoc de Bonferroni e Teste U de Mann-Whitney, considerando o nível de significância de $\mathrm{p}<0,05$. Os resultados mostraram que os meninos obtiveram melhor agilidade e foram mais fisicamente ativos do que as meninas. Do total de participantes, 13 apresentaram sobrepeso, e 17 foram classificados como sedentários. Não houve diferenças na agilidade considerando a classificação do índice de massa corporal ou nível de atividade física. Conclui-se que meninos são mais ágeis e praticam mais atividade física do que meninas. Todavia, a agilidade não apresentou associação com o índice de massa corporal nem com o nível de atividade física
\end{abstract}

Palavras-chave: Atividade física; Aptidão física; Índice de massa corporal; Adolescentes.

\begin{abstract}
The research aimed to verify the association between the level of physical activity, body mass index and the agility of private school adolescents in the city of Senhor do Bonfim/BA. This is a cross-sectional study, with a sample of 75 students ( 35 girls and 40 boys), aged between 10 and 14 years. The data collection included measurements of body mass and stature (for body mass index calculation), a weekly physical activity level questionnaire and an agility test (square test). The normality of data was verified through Kolmogorov-Smirnov's test. Data was analyzed using Student's t-test, One Way ANOVA with Bonferroni's post hoc, and Mann-Whitney's U Test, considering a significance level of $\mathrm{p}<0,05$. The results showed that boys presented better results in agility and physical activity level when compared to girls. Of the total of participants, 13 were overweight and 17 were considered sedentary. No differences were found in agility according to body mass index and physical activity level classification. It is concluded that boys are more agile and more physically active than girls. However, agility was not associated to body mass index nor physical activity level.
\end{abstract}

Keywords: Motor activity; Physical fitness; Body mass index; Adolescents. 


\section{INTRODUÇ̃̃̃O}

A Organização Mundial da Saúde (OMS) define a atividade física como sendo qualquer movimento corporal produzido pelos músculos esqueléticos que requeiram gasto de energia. Entre estes movimentos, incluem-se atividades praticadas no trabalho, execução de tarefas domésticas, jogos, viagens e atividades de lazer ${ }^{1}$. Todavia, mudanças ambientais e sociais têm contribuído para um declínio no nível de atividade física (NAF) de crianças e adolescentes ${ }^{2}$, e essas condições têm sido associadas como aumento da obesidade ${ }^{3,4}$.

Além disso, um baixo NAF durante a infância e a adolescência reflete em um declínio da aptidão física ${ }^{5}$, que é definida como um estado de bem-estar, que se refere à capacidade de realizar tarefas diárias, esportes ou ocupações sem fadiga indevida ${ }^{6}$. A relação entre o excesso de peso e a aptidão física relacionada à saúde tem sido abordada em estudos prévios ${ }^{7-10}$.

No entanto, a aptidão física pode ser classificada de duas formas, sendo: 1) aptidão física relacionada à saúde: envolve variáveis como aptidão cardiorrespiratória, flexibilidade e resistência muscular localizada; e 2) aptidão física relacionada ao desempenho/esporte (força explosiva de membros superiores e inferiores, velocidade, agilidade e, novamente, aptidão cardiorrespiratória) ${ }^{11}$.

Nesse cenário, a agilidade é caracterizada pela capacidade de alterar a direção do movimento rapidamente e está relacionada com o desempenho, principalmente nos esportes ${ }^{12}$. Apesar de a relação entre a agilidade, o NAF e o índice de massa corporal (IMC) não estar bem definida na literatura, sabe-se que a prática regular de exercício físico contribui para a melhoria das aptidões físicas ${ }^{13}$. Ademais, a $\mathrm{OMS}^{1}$ sugere que crianças e adolescentes precisam melhorar capacidades físicas relacionadas à saúde e ao desempenho.
A agilidade também tem sido associada a fatores intervenientes ao cenário esportivo, como melhor cognição e poder de decisão ${ }^{12}$. Ainda, foram observadas associações negativas entre o IMC, o equilíbrio e a agilidade de adolescentes ${ }^{14}$. Dessa forma, esta pesquisa teve como objetivo verificar a relação entre o NAF, a aptidão física (por meio da agilidade) e a classificação do IMC em adolescentes escolares de 10 a 14 anos.

\section{MÉTODOS}

Delineamento do estudo

Trata-se de um estudo de natureza transversal e abordagem quantitativa-descritiva, que se caracterizou por analisar as características de fatos associados e/ou isolamento de variáveis principais ou chave.

\section{Local e característica dos participantes}

O projeto aconteceu no Centro Educacional Edneilda Marques, na cidade de Senhor do Bonfim/BA. Para recrutamento dos participantes, foi divulgado, nas salas, murais e espaços coletivos, o convite à participação em uma reunião de apresentação do projeto. A escola tem dependência administrativa privada, atende alunos do ensino fundamental I e II ( $\left(1^{\circ}\right.$ ao $9^{\circ}$ ano $)$ e possui cerca de 224 alunos matriculados.

Após a explicação do estudo, foi entregue aos alunos o Termo de Consentimento Livre e Esclarecido (TCLE) que deveria ser assinado pelos pais ou responsáveis. Os critérios de inclusão do presente estudo foram: entregar o TCLE devidamente assinado e estar matriculado em alguma turma entre o $6^{\circ}$ e o $9^{\circ}$ ano do ensino fundamental II. 


\section{Procedimentos e instrumentos de coleta}

Para a coleta de dados, os participantes tiveram medidas a sua massa corporal, sua estatura, responderam a um questionário de NAF e realizaram o teste de agilidade. A coleta de dados foi realizada em três dias.

A mensuração da massa corporal foi realizada por meio de uma balança digital com precisão de 100 g. Já a estatura foi medida utilizando uma fita métrica acoplada à parede com precisão de $1 \mathrm{~cm}$. Para ambas as medidas, os participantes trajavam roupas leves e estavam descalços. O IMC foi calculado a partir da divisão da massa corporal $(\mathrm{kg})$ pela estatura elevada à segunda potência $\left(\mathrm{m}^{2}\right)$. Os pontos de corte de IMC utilizados foram os preconizados por Gaya ${ }^{11}$.

O NAF foi estimado a partir do questionário elaborado por Militão et al. ${ }^{15}$, o qual estima o gasto energético (MET) por semana a partir do relato de atividades esportivas, de lazer, de deslocamento e na escola, considerando a frequência, a duração e a intensidade. A classificação dos participantes seguiu o preconizado pelo mesmo estudo, no qual indivíduos com atividade física total menor do que 600 MET $\square$ sem-1 foram caracterizados como sedentários; entre 600 e 1.500 MET $\square$ sem-1, como irregularmente ativos; 1.500 a 2.999 MET $\square$ sem-1, como ativos; e acima de 3.000 MET $\square$ sem-1, como muito ativos.

A agilidade dos participantes foi testada pelo teste do quadrado conforme descrito por Gaya11. Brevemente, um quadrado com quatro metros de lado foi demarcado com cones, e os participantes, ao sinal do avaliador, deslocaram-se até tocar no cone à sua diagonal; em seguida, dirigiram-se ao cone à sua esquerda; posteriormente, cruzaram o quadrado em diagonal novamente; e, por fim, dirigiram-se ao último cone (correspondente ao ponto de partida).

\section{Análise dos dados}

Inicialmente, foi realizada uma análise descritiva dos dados, por meio de média, desvio-padrão, frequência relativa e absoluta, mediana e intervalo interquartil. Após isso, a normalidade dos dados foi testada pelo teste de Kolmogorov Smirnov, no qual foi observado que o NAF não apresentou distribuição normal. Dessa forma, os dados foram analisados a partir de testes paramétricos (Teste-t de Student e ANOVA de uma entrada com post hoc de Bonferroni) e não paramétricos (Teste $\mathrm{U}$ de Mann-Whitney). O nível de significância adotado foi $\mathrm{p}<0,05$, e o software utilizado para a análise dos dados foi o SPSS versão 22.0 para Windows.

O presente estudo encontra-se de acordo com a resolução 466/12 do Conselho Nacional de Saúde e foi aprovado pelo Comitê de Ética em Pesquisa da Universidade Federal do Vale do São Francisco (Univasf) sob o protocolo número $0005 / 220115$.

\section{RESULTADOS}

Participaram do estudo 75 estudantes, sendo 35 meninas e 40 meninos, com idades entre 10 e 14 anos. As características gerais dos participantes podem ser visualizadas na Tabela 1.

Tabela 1. Características gerais dos participantes $(n=75)$

\begin{tabular}{ll}
\hline & Média \pm DP \\
\hline Idade (anos) & $11,91 \pm 0,84$ \\
Massa corporal $(\mathrm{kg})$ & $50,59 \pm 10,69$ \\
Estatura $(\mathrm{m})$ & $1,59 \pm 0,90$ \\
Índice de massa corporal (kg.m-2) & $19,80 \pm 2,99$ \\
Agilidade (seg) & $7,60 \pm 0,72$ \\
Nível de atividade física (MET.sem-1) & $1520,08 \pm 1272,02$ \\
\hline
\end{tabular}


A Tabela 2 apresenta a compara-

estatisticamente significativas, conforme pode ser observado na Tabela 3.

ção das variáveis de acordo com o sexo. Nesse sentido, o Teste-t de Student para amostras independentes revelou diferenças significativas para o teste de agilidade, em que os meninos apresentaram melhores resultados do que as meninas. Ainda, o Teste U de Mann-Whitney mostrou que os meninos foram mais fisicamente ativos

Tabela 2. Comparação das variáveis de acordo com o sexo.

\begin{tabular}{|c|c|c|c|}
\hline & Meninas $(n=35)$ & Meninos $(n=40)$ & $p$ \\
\hline Idade (anos) & $11,91 \pm 0,88$ & $11,90 \pm 0,81$ & 0,942 \\
\hline M. Corporal (kg) & $50,06 \pm 8,62$ & $51,05 \pm 12,60$ & 0,689 \\
\hline Estatura (m) & $1,58 \pm 0,68$ & $1,59 \pm 0,10$ & 0,692 \\
\hline IMC $\left(\mathrm{kg} \cdot \mathrm{m}^{-2}\right)$ & $19,86 \pm 3,01$ & $19,77 \pm 3,02$ & 0,923 \\
\hline Agilidade (seg) & $7,79 \pm 0,62$ & $7,43 \pm 0,78$ & 0,030 \\
\hline NAF (MET.sem-1) & $\begin{array}{c}858,00 \\
(429,00-1102,50)\end{array}$ & $\begin{array}{c}1635,75 \\
(927,62-2654,25)\end{array}$ & 0,000 \\
\hline
\end{tabular}

M. Corporal=massa corporal; IMC=índice de massa |corporal; NAF=nível de atividade física. Dados expressos em média \pm desvio-padrão ou mediana (intervalo interquartil)

No que se refere à classificação do IMC, 62 (82,7\%) participantes apresentaram valores normal para o sexo e a idade, enquanto $13(17,3 \%)$ apresentaram sobrepeso. As comparações da agilidade e do NAF, de acordo com a classificação do IMC, não revelaram diferenças

Tabela 3. Agilidade e nível de atividade física de acordo com a classificação do índice de massa corporal.

\begin{tabular}{|c|c|c|c|}
\hline & Normal $(n=62)$ & Elevado (n=13) & $\mathbf{P}$ \\
\hline Agilidade (seg) & $7,54 \pm 0,73$ & $7,86 \pm 0,68$ & 0,161 \\
\hline NAF (MET-sem-1) & $\begin{array}{c}1057,00 \\
(604,87-2152,50)\end{array}$ & $\begin{array}{c}1023,00 \\
(823,50-1828,75)\end{array}$ & 0,989 \\
\hline
\end{tabular}

$\mathrm{NAF=nível} \mathrm{de} \mathrm{atividade} \mathrm{física.} \mathrm{Dados} \mathrm{expressos} \mathrm{em} \mathrm{média} \pm$ desvio-padrão ou mediana (intervalo interquartil) 
A classificação do NAF revelou que 17 participantes $(22,7 \%)$ eram sedentários; 28 (37,3\%), fisicamente ativos; 22 $(29,3 \%)$, ativos; e apenas $8(10,7 \%)$, muito ativos. A ANOVA de uma entrada com post hoc de Bonferroni não revelou diferenças significativas entre o IMC e a agilidade de acordo com o NAF, conforme Tabela 4.
Por fim, a classificação do teste de agilidade mostrou que 65 voluntários $(86,7 \%)$ apresentaram desempenho fraco, $5(6,7 \%)$ obtiveram desempenho razoável, $3(4,0 \%)$ foram classificados com desempenho bom, e $2(2,7 \%)$, com desempenho muito bom.

Tabela 4. Agilidade e índice de massa corporal de acordo com a classificação do nível de atividade física. Dados expressos em média \pm desvio-padrão

\begin{tabular}{lccccc}
\hline & $\begin{array}{c}\text { Sedentário } \\
(\mathbf{n}=\mathbf{1 7})\end{array}$ & $\begin{array}{c}\text { Irreg. Ativo } \\
(\mathbf{n}=\mathbf{2 8})\end{array}$ & $\begin{array}{c}\text { Ativo } \\
(\mathbf{n = 2 2})\end{array}$ & $\begin{array}{c}\text { Muito Ativo } \\
(\mathbf{n}=\mathbf{8})\end{array}$ & $\mathbf{P}$ \\
\hline Agilidade (seg) & $7,66 \pm 0,78$ & $7,61 \pm 0,67$ & $7,60 \pm 0,74$ & $7,41 \pm 0,85$ & 0,214 \\
IMC (kg.m $\left.{ }^{-2}\right)$ & $19,47 \pm 3,23$ & $20,32 \pm 3,30$ & $20,07 \pm 2,35$ & $17,89 \pm 2,54$ & 0,880
\end{tabular}

IMC=índice de massa corporal; Dados expressos em média \pm desvio-padrão

\section{DISCUSSÃO}

Os resultados do presente estudo revelaram que mais da metade dos participantes eram sedentários ou insuficientemente ativos. Além disso, 86,7\% apresentaram resultado fraco no teste de agilidade, e $17,3 \%$ foram classificados com excesso de peso. Nas comparações de acordo com o sexo, os meninos apresentaram melhor agilidade e maior NAF quando comparados às meninas. Todavia, não foram observadas diferenças significativas entre a agilidade e o NAF, quando comparados de acordo com a classificação do IMC, nem entre a agilidade e o IMC, quando comparados de acordo com a classificação do NAF.

No que se refere ao NAF de escolares, resultados semelhantes têm sido reportados na literatura. Borgoni et al.16 e Da Silveira et al. ${ }^{17}$, estudando uma amostra com perfil semelhante ao do presente estudo, revelaram que $55,1 \%$ e $69,4 \%$, respectivamente, dos escolares foram classificados como sedentários. Segundo Regis et al. ${ }^{18}$, a mudança no comportamento de crianças e adolescentes, especialmente nos horários de lazer, pode ser considerada um fator que explica a diminuição do NAF. De acordo com os autores, verificou-se que grande parte do tempo livre dos participantes era dedicado ao lazer passivo, como assistir TV, jogar videogame e utilizar o celular e o computador.

Dados da última Pesquisa Nacional de Saúde do Escolar (PeNSE), um levantamento nacional realizado pelo Instituto Brasileiro de Geografia e Estatística (IBGE), revelaram que aproximadamente $60 \%$ dos escolares do $9^{\circ}$ ano apresentavam o hábito de assistir mais de duas horas de televisão por dia. Além disso, de acordo com a pesquisa, quando perguntados sobre o tempo que ficavam sentados em um dia da semana (assistindo TV, jogando videogame, ou outras atividades sentados), $56,1 \%$ dos participantes informaram ficar mais de três horas 19 .

O excesso de peso em escolares também tem sido objeto de estudo de diversas pesquisas. Luciano et al. ${ }^{20}$ encontraram uma prevalência de $26,8 \%$ de obesidade em estudantes de 9 a 11 anos de idade. Lima et al. ${ }^{21}$, ao avaliar 583 adolescentes de 11 a 17 anos da cidade de Criciúma - SC, reportaram 33,6\% de excesso de peso entre os participantes. Já na região Norte, Farias et al. ${ }^{22}$ encontraram $27,1 \%$ de excesso de peso ao avaliar 4.165 escolares com idades entre 9 e 18 anos. 
O aumento da prevalência de excesso de peso em crianças e adolescentes, nas últimas décadas, é um fenômeno bem conhecido. Em um estudo de revisão, Pate et al. ${ }^{23}$ relataram que o excesso de peso em crianças e adolescentes está associado ao comportamento sedentário, hábitos alimentares, biomarcadores fisiológicos e à ausência de um ambiente propício para a prática de atividades físicas próximo à residência. Sigmund et al. ${ }^{24}$ também ressaltam a importância que os pais possuem no controle da massa corporal dos filhos. De acordo com os autores, crianças e adolescentes com mães sobrepesadas possuíam chances aumentadas de apresentar excesso de peso. Adicionalmente, filhos de pais fisicamente ativos eram significativamente menos propensos a apresentar sobrepeso ou obesidade.

Os resultados referentes à agilidade dos participantes revelaram que $86,7 \%$ foram classificados como fracos. Esses achados são preocupantes, visto que a agilidade tem sido associada à melhor cognição e poder de decisão ${ }^{12}$. Ademais, além do contexto esportivo, a capacidade de realizar mudanças rápidas de direção pode contribuir para a realização de tarefas realizadas no dia a dia de lazer de crianças e adolescentes ${ }^{25}$.

As comparações entre sexo, por sua vez, demonstraram que os meninos apresentaram melhor agilidade e maior $\mathrm{NAF}^{25-28}$. Esses resultados estão bem documentados na literatura e concordam que, na faixa etária estudada, meninos apresentam melhor aptidão física do que meninas. As principais explicações para esses achados envolvem fatores biológicos (como aumento da circulação de testosterona e hormônio do crescimento, que resultam no aumento da força muscular $)^{25,29}$ e culturais (maior enfrentamento de barreiras e menor para prática de atividade física ${ }^{30} \mathrm{e}$ direcionamento para atividades familiares e de expressão) $)^{31}$.

Outro aspecto abordado na presente investigação foi a comparação da agilidade e NAF de acordo com a classificação do IMC dos participantes, e a comparação entre a agilidade e o IMC de acordo com a classificação do NAF. No presente estudo, não foram observadas diferenças significativas para essas análises. Esses resultados não corroboram a literatura, visto que estudos apontam uma menor agilidade em escolares com maior massa corporal e menor NAF e, adicionalmente, existe uma associação inversa no que tange à prática de atividade física e o IMC ${ }^{14,16-18}$. As inconsistências entre os resultados do presente estudo e as informações disponíveis na literatura podem ser explicadas pela pequena quantidade de participantes, por a amostra ser de conveniência (apenas uma escola) e pertencer a uma faixa etária específica. Nesse sentido, sugere-se que estudos com maior quantidade de participantes e pertencentes a uma faixa etária mais ampla sejam conduzidos para explicar melhor a associação entre as variáveis estudadas.

Mesmo consideradas as limitações deste estudo (expostas acima), os resultados aqui encontrados apresentam importância prática para o acompanhamento da saúde de escolares. Além disso, as informações aqui divulgadas reforçam a necessidade de avaliações periódicas de parâmetros antropométricos e de aptidão física e de melhor conhecimento acerca dos hábitos de lazer (incluindo prática de atividade física no ambiente extracurricular) dessa população; pois, embora a promoção de saúde envolva múltiplos fatores, há evidências de que crianças e adolescentes fisicamente ativos têm maiores probabilidades perpetuarem este comportamento na vida adulta ${ }^{27}$.

\section{CONCLUSÃO}

Considerando os resultados encontrados no estudo, pode-se concluir que meninos apresentam maior agilidade e NAF quando comparados às meninas. Além disso, chama-se a atenção para a prevalência de escolares classificados com sobrepeso, baixo NAF e rendimento fraco na capacidade física agilidade. 


\section{REFERÊNCIAS}

1. World Health Organization (WHO). Global recommendations on physical activity for health. Geneva: World Health Organization; 2010.

2. Drenowatz C, Greier K, Ruedl G, Kopp. Association between club sports participation and physical fitness across 6- to 14-year-old Austrian youth. Int J Environ Res Public Health. 2019;16(18):3392.

3. World Health Organization (WHO). Committee report on the end of childhood obesity. Geneva: World Health Organization; 2016.

4. Sallis JF, Bull F, Guthold R, Heath GW, Inoue $\mathrm{S}$, Kelly $\mathrm{P}$, et al. Progress in physical activity over the Olympic quadrennium. Lancet, 2016;388(10051):1325-36.

5. Fraser BJ, Blizzard L, Tomkinson GR, Lycett K, Wake M, Burgner D, et al. The big leap backward: changes in the jumping performance of Australian children aged 11 - 12-years between 1985 and 2015. J Sports Sci. 2019;37:748-54.

6. Bianco A, Jemni M, Thomas E, Patti A, Paoli A, Ramos RJ, et al. A systematic review to determine reliability and usefulness of the field-based test batteries for the assessment of physical fitness in adolescents - The ASSO project. Int J Occup Med Environ Health, 2015;28:445-478.

7. Yi X, You F, Burns RD, Bai Y, Zhang P. Body mass index and physical fitness among Chinese adolescents from Shandong Province: A cross-sectional study. BMC Public Health. 2019;19(81).

8. Wedell-Neergaard AS, Eriksen L, Grønbæk M, Pedersen BK, Krogh-Madsen R, Tolstrup J. Low fitness is associated with abdominal adiposity and low-grade inflammation independent of BMI. PLoS ONE, 2018;13:e190645.
9. Huang YC, Malina RM. Body mass index and individual fitness tests in Taiwanese youth aged 9-18 years. Int J Pediatr Obes. 2010;5:404-11.

10. Gonzalez-Suarez CB, Caralipio N, Gambito E, Reyes JJ, Espino RV, Macatangay R. The association of physical fitness with body mass index and waist circumference in Filipino preadolescents. Asia Pac J Public Health. 2013;25:74-83.

11. Gaya A, Lemos A, Gaya A, Teixeira D, Pinheiro E, Moreira R. Projeto esporte Brasil: manual de testes e avaliação. Porto Alegre: UFRGS; 2016.

12. Horička P, Šimonek J, Paška L. Relationship between reactive agility, cognitive abilities, and intelligence in adolescents. $\mathrm{J}$ Phys Educ Sport. 2020;304:2263-68.

13. Elbe AM, Wikman JM, Zheng M, Larsen MN, Nielsen G, Krustrup P. The importance of cohesion and enjoyment for the fitness improvement of 8-10-year-old children participation in a team and individual sport school-based physical activity intervention. Eur J Sport Sci. 2017;17:343-50.

14. Mughal AW, Shah I, Kan AQ. Correlation of body mass index with selected physical fitness parameters among adolescents of Khyber Pakhtunkhwa, Pakis$\tan$. The Spark a Hec Recognized Journal. 2020;4(1):99-104.

15. Militão AG, Silva, FRD, Peçanha LM, Souza JWS, Militão ESG, Campbell CSG. Reprodutibilidade e validade de um questionário de avaliação do nível de atividade física e comportamento sedentário de escolares de 10 a 13 anos de idade, Distrito Federal, Brasil, 2012. Epidemiol Serv Saúde. 2013;22(1):111-20.

16. Borgoni AP, Ferrari HR, Silva LG, Costa JLD, Dias CP, Tiggemann CL. Perfil da aptidão física, do estado nutricional e do nível de atividade física de crianças de escolas municipais da cidade de Venâncio Aires-RS. Destaques Acadêmicos, Lajeado. 2017;9(3):137-46.

17. Da Silveira BA, Kunrath CA, Dias 
CP, Tiggemann CL. Avaliação do perfil nutricional e prática de atividade física de alunos de séries iniciais do ensino público e privado dos municípios de Encantado e Vespasiano Côrrea/RS. Destaques Acadêmicos, Lajeado. 2015;7(3):160-66.

18. Regis MF, Oliveira LMFT, Santos ARM, Leonildo ACR, Diniz PRB, Freitas CMSM. Urban versus rural lifestyle in adolescents: associations between environment, physical activity levels and sedentary behavior. Einstein (São Paulo). 2016;14(4):461-67.

19. Instituto Brasileiro de Geografia e Estatística (IBGE). Pesquisa Nacional de Saúde do Escolar 2015. Rio de Janeiro: IBGE; 2016.

20. Luciano AP, Bertoli CJ, Adami F, De Abreu LC. Nível de atividade física em adolescentes saudáveis. Rev Brasileira Medicina Esporte. 2016;22(3).

21. Lima TR, Moraes MS, Andrade JHC, Farias JM, Silva DAS. Fatores associados à presença isolada e simultânea de excesso de peso e obesidade abdominal em adolescentes. Rev Paul Pediatr. 2020;38:e2018332.

22. Farias ES, Moreira KFA, Satnos JP, Gemelli IFB, Costa GM, Souza OF. Sobrepeso e obesidade: prevalência em crianças e adolescentes ao Norte do Brasil. J hum Growth Dev. 2020;30(2):266-73.

23. Pate RR, O'Neill JR, Liese AD, Janz KF, Granberg EM, Colabianchi N, et al. Factors associated with development of excessive fatness in children and adolescents: a review of prospective studies. Obes Rev. 2013;14:645-58.

24. Sigmund E, Sigmundová D, Badura P. Excessive body weight of children and adolescents in the spotlight of their parents' overweight and obesity, physical education, and screen time. Int J Public Health. 2020;1-9.

25. Lloyd RS, Read P, Oliver JL, Meyers RW, Nimphius S. Considerations for the development of agility during childhood and adolescence. J Strength Cond Res. 2013;25(3):2-11.
26. Joaquim AG, Dos Santos AR, Rosa LF, Correlação entre nível de flexibilidade $\mathrm{e}$ desempenho na agilidade em escolares de 07 a 10 anos: um estudo transversal. RBPFEX. 2017;11(71)(supl. 2):997-1005.

27. Dumith SC, Ramires VV, Souza MJA, Moraes DS, Petry FG, Oliveira ES, et al. Aptidão física relacionada ao desempenho motor em escolares de sete a 15 anos. Rev Bras Educ Fís Esporte. 2010;24:5-14.

28. Sacchetti R, Ceciliani A, Garulli A, Masotti A, Poletti G, Beltramini P, et al. Physical fitness of primary school children in relation to overweight prevalence and physical activity habits. J Sports Sci. 2012;30(7):633-40.

29. Guessogo WR, Mekoulou-Ndongo J, Assomo-Ndemba PB, Hamadon A, Biassi O, Tsogbny-Panka C, et al. Gender differences in physical fitness among Cameroonian school children aged 10 to 15 in Yaounde City. Int. J School Health. 2020;7(3):45-54.

30. Portela-Pino I, López-Castedo A, Martínez-Patiño MJ, Valverde-Esteve T, Domínguez-Alonso J. Gender differences in motivation and barriers for the practice of physical exercise in adolescence. Int J Environ Res Public Health. 2020;17(1):168.

31. Seabra AF, Mendonça DM, Thomis MA, Anjos LA, Maia JA. Determinantes biológicos e sócio-culturais associados à prática de atividade física de adolescentes. Cad Saúde Pública. 2008;24(4):721-36.
Recebido: 07/12/2019

Aprovado: 14/09/2020 